\title{
National Institute for Health and Clinical Excellence
}

Issue date: July 2007

Review date: May 2010

\section{Varenicline for smoking cessation}

NICE technology appraisal guidance 123 
NICE technology appraisal guidance 123

Varenicline for smoking cessation

\section{Ordering information}

You can download the following documents from www.nice.org.uk/TA123

- $\quad$ The full guidance (this document).

- $\quad$ A quick reference guide for healthcare professionals.

- Information for people who want to quit smoking and their carers ('Understanding NICE guidance').

- Details of all the evidence that was looked at and other background information.

For printed copies of the quick reference guide or 'Understanding NICE guidance', phone the NHS Response Line on 08701555455 and quote:

- $\quad \mathrm{N} 1284$ (quick reference guide)

- $\quad$ N1285 ('Understanding NICE guidance').

\section{This guidance is written in the following context}

This guidance represents the view of the Institute, which was arrived at after careful consideration of the evidence available. Healthcare professionals are expected to take it fully into account when exercising their clinical judgement. The guidance does not, however, override the individual responsibility of healthcare professionals to make decisions appropriate to the circumstances of the individual patient, in consultation with the patient and/or guardian or carer.

\section{National Institute for Health and Clinical Excellence}

MidCity Place

71 High Holborn

London

WC1V 6NA

www.nice.org.uk

(C) National Institute for Health and Clinical Excellence, July 2007. All rights reserved. This material may be freely reproduced for educational and not-for-profit purposes. No reproduction by or for commercial organisations, or for commercial purposes, is allowed without the express written permission of the Institute. 


\section{Contents}

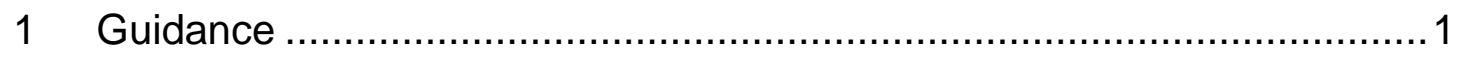

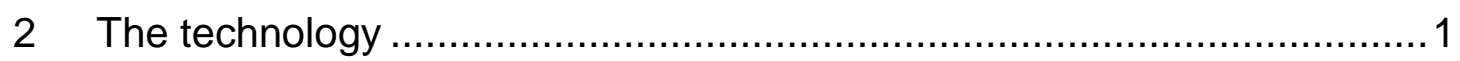

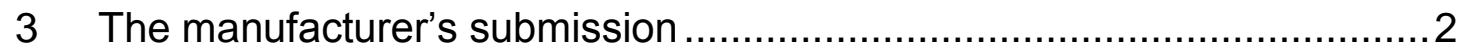

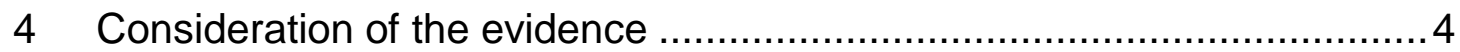

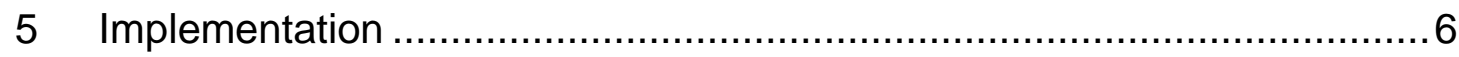

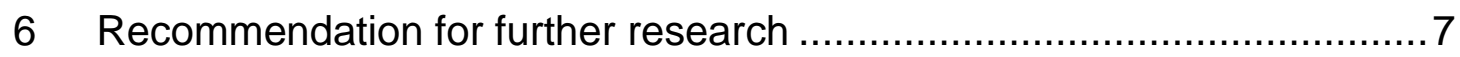

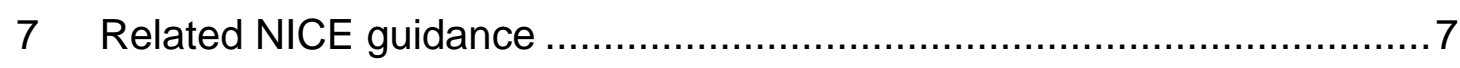

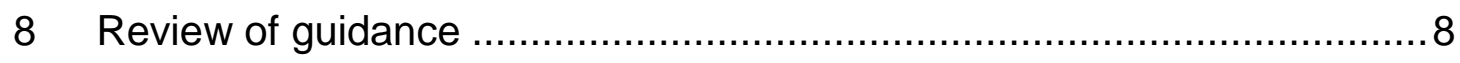

Appendix A. Appraisal Committee members, public health programme

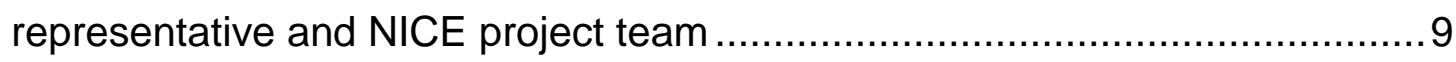

Appendix B. Sources of evidence considered by the Committee.................. 12 


\section{Guidance}

1.1 Varenicline is recommended within its licensed indications as an option for smokers who have expressed a desire to quit smoking.

1.2 Varenicline should normally be prescribed only as part of a programme of behavioural support.

\section{The technology}

2.1 Varenicline (Champix, Pfizer) has marketing authorisation for smoking cessation in adults. The summary of product characteristics (SPC) states that smokers should set a date to stop smoking and treatment with varenicline should start 1 to 2 weeks before this date and that smoking cessation therapies are more likely to succeed for patients who are provided with additional advice and support.

2.2 Varenicline binds with high affinity and selectivity at the $\alpha 4 \beta 2$ neuronal nicotinic acetylcholine receptor, where it acts as a partial agonist. Its binding both alleviates symptoms of craving and withdrawal, and reduces the rewarding and reinforcing effects of smoking by preventing nicotine binding to $\alpha 4 \beta 2$ receptors.

2.3 Varenicline may be associated with nausea and other gastrointestinal disorders such as vomiting. For full details of side effects and contraindications, see the SPC.

2.4 Varenicline is available in $0.5-\mathrm{mg}$ and $1-\mathrm{mg}$ film-coated tablets. The cost is $£ 54.60$ for a 56 -blister pack of 0.5 - or 1 -mg tablets ('British national formulary' [BNF] edition 53). A 12-week course of treatment costs about £163.80. The SPC specifies the option of an additional 12 weeks of treatment and the consideration of dose tapering. Costs may vary in different settings because of negotiated procurement discounts. 
The Appraisal Committee (appendix A) considered evidence submitted by the manufacturer of varenicline and a review of this submission by the Evidence Review Group (ERG: appendix B).

3.1 The manufacturer's primary analysis compared the standard 12week course of varenicline with bupropion and nicotine replacement therapy (NRT). The manufacturer identified four randomised controlled trials (RCTs). Two were three-arm trials that compared varenicline, bupropion and placebo $(n=1483$ and 1413). Another trial compared maintenance treatment (24-week course of varenicline) with placebo $(n=2416)$. The manufacturer also presented data from an open-label trial $(n=957)$ that compared varenicline with NRT.

3.2 The two trials that compared varenicline and bupropion showed that the continuous quit rate for weeks 9-12 was statistically significantly greater for varenicline: odds ratio (OR) $1.93(95 \% \mathrm{Cl}$ 1.40 to 2.68$)$ and OR 1.90 (95\% Cl 1.40 to 2.68), respectively. Both trials also showed that the continuous quit rate for varenicline was statistically significantly greater than for placebo: OR $3.85(95 \% \mathrm{Cl}$ 2.70 to 5.50$)$ and $\mathrm{OR} 3.85(95 \% \mathrm{Cl} 2.69$ to 5.50), respectively. For the longer time horizon of weeks 9-52, the ORs for varenicline compared with bupropion, respectively for the two trials, were 1.46 (95\% Cl 0.99 to 2.17) and 1.77 (95\% Cl 1.19 to 2.63). The maintenance trial that compared 24-week varenicline with placebo showed that the continuous quit rate was statistically significantly greater with varenicline than with placebo: weeks 13-24 OR 2.47 (95\% Cl 1.95 to 3.15$)$; weeks $13-52$ OR 1.35 (95\% Cl 1.07 to 1.70). The results of the open-label trial were marked confidential by the manufacturer.

3.3 The manufacturer also submitted a meta-analysis of 70 NRT trials, 12 bupropion trials and 4 varenicline trials against control/placebo. 
The meta-analysis indirectly compared the efficacy of the treatments based on relative treatment effects. This indirect comparison showed that at 12 months varenicline was superior to NRT (OR 1.66 [Cl 1.17 to 2.36]) and bupropion (OR 1.58 [95\% Cl $1.22-2.05])$. Varenicline was also superior at 3 months to both NRT (OR 1.78 [95\% Cl 1.23 to 2.57]) and bupropion (OR 1.61 [95\% Cl 1.17 to 2.22$]$ ).

3.4 The manufacturer presented a cost-effectiveness analysis based on a Markov model. It assumes an individual makes a single quit attempt at the beginning of the model. The individual is followed from this initial quit attempt to various health states and potential comorbidities including lung cancer, asthma exacerbations, chronic obstructive pulmonary disease, stroke and cardiovascular disease. The probabilities of relapsing and developing comorbidities are assumed to decrease over time from smoking cessation. The efficacy rates for the treatments are calculated from the odds ratios derived from the results of the pooled direct clinical trials and the indirect comparison. The probabilities associated with relapse are derived from relative risks reported in US-based long-term longitudinal and cohort studies into smoking and abstinence. The costs and utilities are derived from several published sources. Some health-related utility estimates are based on US data, including baseline health-related utilities.

3.5 The base-case analysis showed that over a lifetime horizon varenicline dominated bupropion and NRT - that is, it was cheaper and more effective. Variation of the time horizon used in the analysis showed that, at 20 years and over, varenicline maintained its dominating position. Sensitivity analyses included altering baseline health-related utilities and costs of NRT, and the use of efficacy rates from the direct open-label trial that compared varenicline with NRT. Over a lifetime horizon varenicline dominated NRT and bupropion in all sensitivity analysis. 
3.6 The ERG noted that the inclusion/exclusion criteria of the metaanalysis in the manufacturer's submission differed from existing analyses by the Cochrane collaboration and considered that they could overestimate the efficacy of varenicline. The ERG also conducted its own meta-analysis and indirect comparison which suggested that the odds ratio for varenicline in comparison with NRT was lower than in the manufacturer's model: OR $1.54(95 \% \mathrm{Cl}$ 1.10 to 2.16$)$.

3.7 The ERG noted that the assumptions included in the model could make external validity questionable. For example, it considered that the assumption of a single quit attempt was a limitation that did not allow consideration of the impact of subsequent quit attempts on costs, morbidity or mortality. In addition, the extrapolation of data on 1-year quit rates to a lifetime is associated with considerable uncertainty surrounding the long-term relapse or abstinence experience of the model cohort. The ERG noted that the use of indirect comparison in the base case was inappropriate given the availability of direct trial evidence. The ERG further identified some computational errors in the calculation of transition probabilities and population calculations. The ERG commented that the method used to convert odds ratios to efficacy rates was not validated and a model constructed around odds ratios would have been more appropriate. The ERG compared the number of life years gained in the model with the results of two published analyses and found no substantial differences.

3.8 Full details of all the evidence are in the manufacturer's submission and the ERG report, which are available from www.nice.org.uk/TA123

\section{Consideration of the evidence}

4.1 The Appraisal Committee reviewed the data available on the clinical and cost effectiveness of varenicline, having considered 
evidence on smoking cessation and the value placed on the benefits of varenicline by people who smoke tobacco products and want to quit, those who represent them, and clinical specialists. It was also mindful of the need to take account of the effective use of $\mathrm{NHS}$ resources.

4.2 The Committee considered the clinical effectiveness evidence presented by the manufacturer. It concluded that the evidence from the direct trials and the systematic reviews carried out by the manufacturer and ERG demonstrated that varenicline was superior to NRT and bupropion in achieving continuous abstinence. The Committee heard from the clinical specialists and patient experts that the success rates with varenicline made it a useful addition to the variety of interventions available in smoking cessation, particularly because many smokers need to make multiple quit attempts. The availability of an additional treatment choice was mentioned by clinical specialists and patient experts as beneficial to those having difficulty maintaining abstinence and avoiding relapse because it enabled them to have more control.

4.3 The Committee considered the evidence on the cost effectiveness of varenicline submitted by the manufacturer. The Committee noted the comments of the ERG that the submission was not transparent and possessed limited external validity. The model included an extrapolation of 1-year clinical data to a lifetime horizon and included an assumption of a single quit attempt. The Committee also noted the computational errors identified by the ERG, and noted that the ERG had expressed concerns about a number of other assumptions in the model, in particular the use of US data for baseline risk and the use of all-cause morbidity instead of othercause morbidity. Nevertheless, the Committee considered that these concerns were not sufficient to undermine the inference that the use of varenicline in smoking cessation was likely to be a costeffective use of NHS resources. 
4.4 The Committee heard from clinical specialists about the importance of counselling and support in smoking cessation to reinforce the commitment required to quit smoking. It noted that varenicline had been provided alongside counselling and support in the clinical trials. However, the Committee also heard from the clinical specialists that counselling and support are not always used by people aiming to stop smoking and that pharmacotherapies can be effective in the absence of such programmes. The Committee concluded that varenicline should normally be provided in conjunction with counselling and support, but that if such support is refused or is not available, this should not preclude treatment with varenicline.

\section{$5 \quad$ Implementation}

5.1 The Healthcare Commission assesses the performance of NHS organisations in meeting core and developmental standards set by the Department of Health in 'Standards for better health' issued in July 2004. The Secretary of State has directed that the NHS provides funding and resources for medicines and treatments that have been recommended by NICE technology appraisals normally within 3 months from the date that NICE publishes the guidance. Core standard C5 states that healthcare organisations should ensure they conform to NICE technology appraisals.

5.2 'Healthcare Standards for Wales' was issued by the Welsh Assembly Government in May 2005 and provides a framework both for self-assessment by healthcare organisations and for external review and investigation by Healthcare Inspectorate Wales. Standard 12a requires healthcare organisations to ensure that patients and service users are provided with effective treatment and care that conforms to NICE technology appraisal guidance. The Assembly Minister for Health and Social Services issued a Direction in October 2003 which requires Local Health Boards and 
NHS Trusts to make funding available to enable the implementation of NICE technology appraisal guidance, normally within 3 months.

5.3 NICE has developed tools to help organisations implement this guidance (listed below). These are available on our website (www.nice.org.uk/TA123).

- Local costing template incorporating a costing report to estimate the savings and costs associated with implementation.

- Audit criteria to monitor local practice.

\section{$6 \quad$ Recommendation for further research}

6.1 The Committee recommends that research is conducted into the long-term effectiveness of smoking cessation interventions with particular reference to relapse rates after completion of treatment.

\section{$7 \quad$ Related NICE guidance}

7.1 NICE has issued the following related technology appraisal guidance and clinical guidelines.

- Guidance on the use of nicotine replacement therapy (NRT) and bupropion for smoking cessation. NICE technology appraisal guidance 39 (2002). Available from: www.nice.org.uk/TA039

- Brief interventions and referral for smoking cessation in primary care and other settings. NICE public health intervention guidance 1 (2006). Available from: www.nice.org.uk/PHI001

- Workplace health promotion: how to help employees to stop smoking. NICE public health intervention guidance 5 (2007). Available from: www.nice.org.uk/PHI005

7.2 NICE is developing the following guidance (details available from www.nice.org.uk) 
- Smoking cessation services, including the use of pharmacotherapies, in primary care, pharmacies, local authorities and workplaces, with particular reference to manual working groups, pregnant women who smoke and hard to reach communities. NICE public health programme guidance (publication expected November 2007).

\section{$8 \quad$ Review of guidance}

8.1 The review date for a technology appraisal refers to the month and year in which the Guidance Executive will consider whether the technology should be reviewed. This decision will be taken in the light of information gathered by the Institute, and in consultation with consultees and commentators.

8.2 The guidance on this technology will be considered for review in May 2010.

Andrew Dillon

Chief Executive

July 2007 


\section{Appendix A. Appraisal Committee members, public health programme representative and NICE project team}

\section{A. Appraisal Committee members}

The Appraisal Committee is a standing advisory committee of the Institute. Its members are appointed for a 3-year term. A list of the Committee members who took part in the discussions for this appraisal appears below. The Appraisal Committee meets three times a month except in December, when there are no meetings. The Committee membership is split into three branches, with a chair and vice chair. Each branch considers its own list of technologies, and ongoing topics are not moved between the branches.

Committee members are asked to declare any interests in the technology to be appraised. If it is considered there is a conflict of interest, the member is excluded from participating further in that appraisal.

The minutes of each Appraisal Committee meeting, which include the names of the members who attended and their declarations of interests, are posted on the NICE website.

\section{Professor David Barnett}

Professor of Clinical Pharmacology, University of Leicester

\section{Dr David W Black}

Director of Public Health, Derbyshire County PCT

\section{Mr Brian Buckley}

Chairman, Incontact

\section{Dr Carol Campbell}

Senior Lecturer, University of Teesside

\section{Professor Mike Campbell}

Professor of Medical Statistics, University of Sheffield 


\section{Ms Jude Cohen}

Manager of Resources and Administration, Council for Psychotherapy (UKCP)

\section{Dr Christine Davey}

Senior Researcher, North Yorkshire Alliance R \& D Unit

\section{Dr Mike Davies}

Consultant Physician, Manchester Royal Infirmary

\section{Mr Richard Devereaux-Phillips}

Public Affairs Manager, Medtronic

Dr Rachel A Elliott

Clinical Senior Lecturer, University of Manchester

\section{Mrs Eleanor Grey}

Lay member

\section{Dr Catherine Jackson}

Clinical Lecturer in Primary Care Medicine, Alyth Health Centre

\section{Dr Peter Jackson}

Clinical Pharmacologist, University of Sheffield

\section{Ms Rachel Lewis}

Practice Development Facilitator, Manchester PCT

\section{Professor Jonathan Michaels}

Professor of Vascular Surgery, University of Sheffield

\section{Dr Eugene Milne}

Deputy Medical Director, North East Strategic Health Authority

\section{Dr Richard Alexander Nakielny}

Consultant Radiologist, Royal Hallamshire Hospital, Sheffield

\section{Dr Katherine Payne}

Health Economics Research Fellow, University of Manchester 


\section{Professor Andrew Stevens (Chair)}

Professor of Public Health, University of Birmingham

\section{Dr Cathryn Thomas}

Senior Lecturer, University of Birmingham

\section{B Public health programme representative}

The following individual, representing the programme development group responsible for developing the Institute's public health programme guidance related to this topic, attended the meeting to observe and to contribute as an adviser to the Committee.

- Dr Paul Aveyard, Senior Lecturer, Department of Primary Care and General Practice, University of Birmingham

\section{NICE project team}

Each technology appraisal is assigned to a team consisting of one or more health technology analysts (who act as technical leads for the appraisal), a technical adviser and a project manager.

Prashanth Kandaswamy

Technical Lead

\section{Louise Longworth}

Technical Adviser

\section{Chris Feinmann}

Project Manager 


\section{Appendix B. Sources of evidence considered by the Committee}

A The evidence review group (ERG) report for this appraisal was prepared by the School of Health and Related Research (ScHARR):

D Hind, P Tappenden, J Peters, K Kenjegalieva. Varenicline for smoking cessation: a single technology appraisal (March 2007).

B The following organisations accepted the invitation to participate in this appraisal. They were invited to comment on the draft scope.

Organisations listed in I were also invited to make written submissions. Organisations listed in II gave their expert views on varenicline by providing a written statement to the Committee. Organisations listed in I and II have the opportunity to appeal against the final appraisal determination.

I Manufacturer/sponsor:

- $\quad$ Pfizer Ltd

II Professional/specialist and patient/carer groups:

- $\quad$ Action Heart

- $\quad$ British Heart Foundation

- Burnley, Pendle and Rossendale PCT

- $\quad$ Cancer Research UK

- $\quad$ General Practice Airways Group

- Macmillan Cancer Relief

- $\quad$ National Lung Cancer Forum for Nurses

- Primary Care Cardiovascular Society

- Roy Castle Lung Cancer Foundation

- Royal College of Nursing

- Royal College of Physicians

- Welsh Assembly Government

III Commentator organisations (did not provide written evidence and without the right of appeal):

- $\quad$ British National Formulary

- $\quad$ British Society for Cardiovascular Research 
- Department of Health, Social Services and Public Safety for Northern Ireland

- $\quad$ GlaxoSmithKline (Bupropion)

- GlaxoSmithKline Consumer Healthcare (Nicotine)

- Medicines and Healthcare Products Regulatory Agency (MHRA)

- National Coordinating Centre for Health Technology Assessment

- $\quad$ NHS Quality Improvement Scotland

School of Health and Related Research (ScHARR), University of Sheffield

C The following individuals were selected from clinical specialist and patient advocate nominations from the professional/specialist and patient/carer groups. They gave their expert personal view on varenicline by providing written evidence to the Committee.

- Dr Katherine Willmer, Consultant Cardiologist, nominated by the British Cardiovascular Society - clinical specialist

- Mrs Christine Owens, Head of Tobacco Control of and nominated by the Roy Castle Lung Cancer Foundation patient expert

- Mr David Geldard, President, Heart Care Partnership (UK), nominated by the British Cardiovascular Society - patient expert 